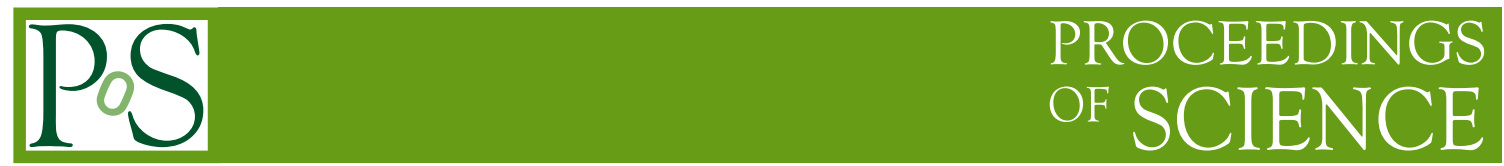

\title{
Recent results on collectivity in small systems from ALICE
}

\author{
Zhanna Khabanova ${ }^{a, *}$ on behalf of the ALICE Collaboration \\ ${ }^{a}$ Nikhef, \\ Science Park 105, Amsterdam, The Netherlands \\ E-mail: zhannak@nikhef.nl
}

Recent measurements performed at the LHC have revealed an unexpected presence of the near-side ridge and other signs of collectivity in $\mathrm{p}-\mathrm{Pb}$ and $\mathrm{pp}$ collisions, referred to as small systems. Since in heavy-ion collisions these effects are caused by the formation and collective expansion of a quark-gluon plasma (QGP), such observations in small systems have triggered the ongoing debate about their origin. In this work, a brief overview of the latest ALICE results in small systems is presented. These include studies of two-particle angular correlations, charge balance functions, anisotropic flow and transverse momentum $\left(p_{\mathrm{T}}\right)$ spectra of identified hadrons.

The Eighth Annual Conference on Large Hadron Collider Physics-LHCP2020

25-30 May, 2020

online

${ }^{*}$ Speaker 


\section{Introduction}

A new, deconfined state of a strongly interacting matter, called quark-gluon plasma (QGP), is produced in the collisions between ultrarelativistic heavy ions at the LHC [1]. Previous studies of the QGP properties allowed one to conclude that its evolution is effectively described by equations of viscous hydrodynamics for a nearly perfect liquid [2]. According to this description, QGP expands with collective averaged transverse velocity, referred to as radial flow. In addition, the hydrodynamic response of the medium to the initial anisotropic geometry of the overlapping region leads to the observation of the final-state anisotropy in momentum space, referred to as anisotropic flow.

Several effects, interpreted as a consequence of the collective expansion in heavy-ion collisions, were also unexpectedly observed in $\mathrm{p}-\mathrm{A}$ and pp collisions. The first hints originated from twoparticle angular correlations measured as a function of $\Delta \eta$ and $\Delta \varphi$, i.e. the relative pseudorapidity and azimuthal angle of each particle pair, respectively. In $\mathrm{Pb}-\mathrm{Pb}$ collisions, anisotropic flow leads to an enhancement in the amount of correlated pairs, located around $\Delta \varphi \sim \pi$ as well as $\Delta \varphi \sim 0$ and extending over a long-range in $\Delta \eta$, with the latter being referred to as the near-side ridge [3]. The discovery of this feature in high-multiplicity $\mathrm{p}-\mathrm{Pb}[4]$ and $\mathrm{pp}$ [5] collisions initiated the debate on whether such behavior originates from the QGP formation or other alternative mechanisms that might lead to similar structures without involving the QGP, such as color correlations computed in the color glass condensate effective field theory [6], elastic parton-parton scattering employed in parton transport models [7] or color reconnection [8].

In these proceedings, a short review of the latest measurements from ALICE experiment with the aim of gaining a better understanding about the origin of collective phenomena in small systems is reported.

\section{Results}

\subsection{Two-particle angular correlations and $\mathbf{v}_{2}(\eta)$}

Experimentally, two-particle angular correlations can be expressed in terms of the associated per-trigger yield defined as:

$$
\frac{1}{N_{\text {trig }}} \frac{d^{2} N_{\text {assoc }}}{d \Delta \eta d \Delta \varphi}=\frac{S(\Delta \eta, \Delta \varphi)}{B(\Delta \eta, \Delta \varphi)},
$$

where $N_{\text {trig }}$ is the number of trigger particles, $S(\Delta \eta, \Delta \varphi)=1 / N_{\text {trig }} d^{2} N_{\text {same }} / d \Delta \eta d \Delta \varphi$ is the signal distribution calculated correlating the trigger particle with the rest of associated particles in the same event, while $B(\Delta \eta, \Delta \varphi)=\alpha d^{2} N_{\text {mixed }} / d \Delta \eta d \Delta \varphi$ is the background distribution obtained using mixed events and applied to account for particle pair acceptance effects. The $\alpha$ factor is used to normalize the mixed event distribution to unity at $(\Delta \eta, \Delta \varphi)=(0,0)$. In previous measurements of associated per-trigger yields performed by ALICE using its central barrel detectors in $\mathrm{Pb}-\mathrm{Pb}[9]$, $\mathrm{p}-\mathrm{Pb}[10]$ and high-multiplicity pp collisions, the near-side ridge was observed up to $\Delta \eta \sim 2$. In order to investigate the evolution of this structure in small systems further, the studies are extended to a wider range of ALICE acceptance using the ALICE Forward Multiplicity Detector (FMD) with the pseudorapidity coverage of $-3.4 \leq \eta \leq-1.7$ (FMD3) and $1.7 \leq \eta \leq 5.0$ (FMD1,2) [11]. 

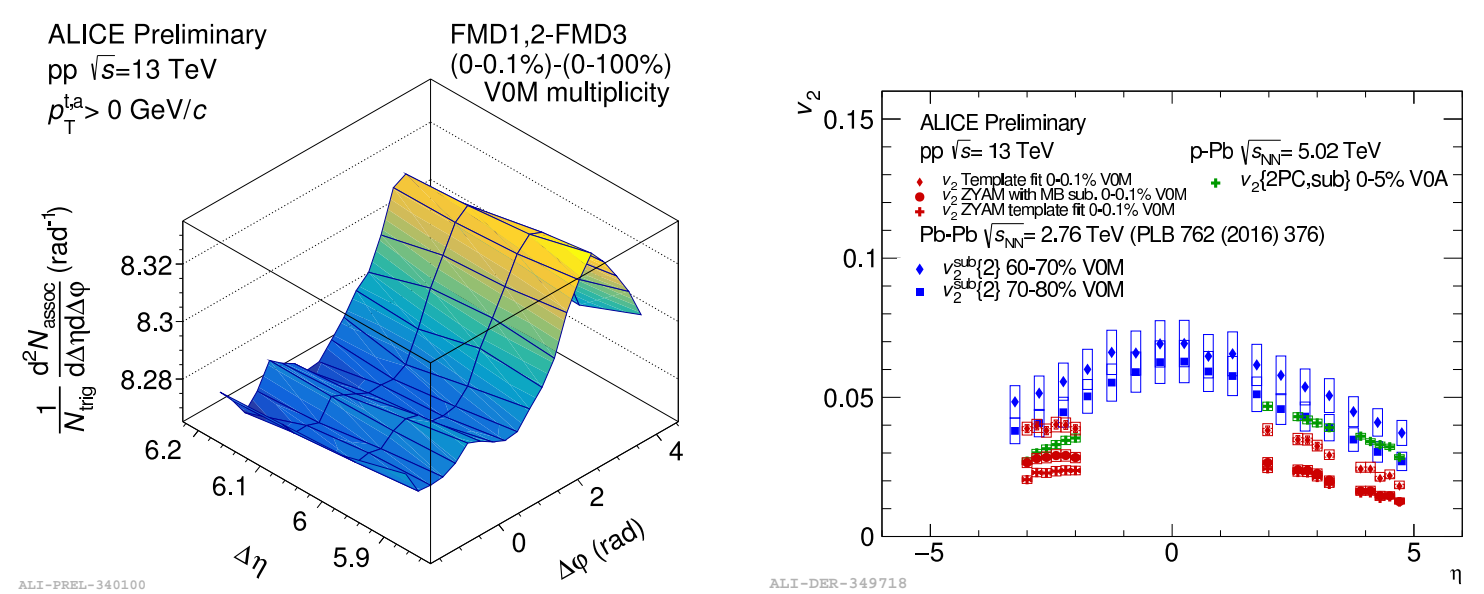

Figure 1: (Left) Associated per-trigger yield for FMD1,2-FMD3 correlations in 0-0.1\% multiplicity class in pp collisions at $\sqrt{s}=13 \mathrm{TeV}$. (Right) $v_{2}(\eta)$ in peripheral $\mathrm{Pb}-\mathrm{Pb}$ and high-multiplicity $\mathrm{p}-\mathrm{Pb}$ and pp collisions, where in the latter case three sets of data points correspond to different non-flow subtraction methods.

The left panel of Fig. 1 shows the resulting associated per-trigger yield for FMD1,2-FMD3 correlations in $0-0.1 \%$ fraction of events with highest multiplicity in pp collisions, corrected for the contribution from non-flow effects by subtracting the minimum bias sample. It can be seen that the near-side ridge persists up to $\Delta \eta \sim 6$. Similar results are obtained in $\mathrm{p}-\mathrm{Pb}$ collisions with a subtraction of the peripheral multiplicity class, according to the procedure developed and proposed in [12], which leads to the observation of the ridge up to $\Delta \eta \sim 8$. In addition, in both systems $v_{2}(\eta)$ is extracted using the projection of the two-dimensional per-trigger yield on $\Delta \varphi$, denoted as $Y^{\text {ridge }}(\Delta \varphi)$, within the long-range part of pseudorapidity values. In $\mathrm{p}-\mathrm{Pb}$ collisions, this is done by fitting $Y^{\text {ridge }}(\Delta \varphi)$ for TPC-FMD3, TPC-FMD1,2 and FMD1,2-FMD3 correlations separately with Fourier series according to $Y^{\text {ridge }}(\Delta \varphi)=a_{0}+2 \sum_{n=1}^{3} a_{i} \cos (n \Delta \varphi)$ and extracting the fit parameter $a_{2}$ that defines the corresponding modulation $V_{2}$. After this, the $v_{2}$ value at a certain $\eta$ bin is

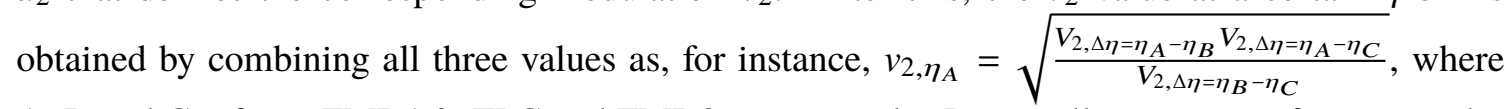
A, B and C refer to FMD1,2, TPC and FMD3, respectively. In pp collisions, apart from a similar procedure, two other methods, applied previously by ATLAS collaborations are used. The first one is based on the template fitting, while the second one relies on the alternative version of template fitting, referred to as ZYAM template fit [13]. The final $v_{2}(\eta)$ in both systems are shown in the right panel of Fig. 1 and are compared to the previous results in peripheral $\mathrm{Pb}-\mathrm{Pb}$ collisions. The asymmetry in $\mathrm{p}-\mathrm{Pb}$ results is due to the difference in charged particle multiplicity as a function of $\eta$ in the p-going and $\mathrm{Pb}$-going directions, while in pp this is caused by a bias from the multiplicity estimator. The data in $0-5 \%$ multiplicity class in $\mathrm{p}-\mathrm{Pb}$ collisions is in agreement with the data in $70-80 \%$ peripheral $\mathrm{Pb}-\mathrm{Pb}$ collisions, where the multiplicities at forward pseudorapidities are close. Future comparison of presented results with model predictions might provide more insight into the origin of long-range two-particle correlations in small systems.

\subsection{Balance function}

Balance function is used to measure the charge-dependent part of two-particle correlations and is calculated as following: 


$$
B(\Delta \eta, \Delta \varphi)=\frac{1}{2}\left[C_{(+,-)}+C_{(-,+)}-C_{(+,+)}-C_{(-,-)}\right],
$$

where $C_{(+,-)}$is the associated per-trigger yield computed for a positive trigger particle and negative associated particles according to Eq. 1, similarly as the rest of terms for other unlike- and like-sign charge combinations. The subtraction of like-sign terms is used to remove same-charge correlations and statistically isolate the charge-dependent part. This leads to the fact that anisotropic flow effects are cancelled out in the balance function measurement. However, the effect of radial flow is still present and affects the balancing charges. In heavy-ion collisions, this is reflected in the narrowing of the balance function width for charged hadrons in central with respect to peripheral events due to an increase in radial flow in the first with respect to the second case [14]. Similar trend has been observed in $\mathrm{p}-\mathrm{Pb}$ and $\mathrm{pp}$ collisions, which is consistent with the idea of collective effects being present in small systems. In addition, a comparison of the data for charged particles in $p p$ collisions to predictions from the PYTHIA8 model [15] with color reconnection shows a qualitative agreement, while the tune without color reconnection is not able to reproduce experimental results. The origin of a multiplicity-dependent narrowing of the width in pp collisions can be investigated better in the analysis for identified hadrons. In this analysis, a more pronounced decrease with increasing multiplicity is expected for heavier particles if radial flow or other effects as color reconnection, mimicking a radial-flow pattern, are the main reason behind this trend due to the direct dependence of transverse momentum on the mass of the particle.

Fig. 2 presents the results of the balance function width as a function of multiplicity class for pion and proton pairs in pp collisions at $\sqrt{s}=5.02 \mathrm{TeV}$, compared to corresponding predictions from PYTHIA8 with and without the color reconnection. Contrary to the aforementioned expectation, a more pronounced narrowing is observed for pions instead of protons in the data. Moreover, neither of PYTHIA8 tunes is able to reproduce the data. These results suggest that there might be other effects that are not connected to collectivity that drive this behavior in pp collisions.
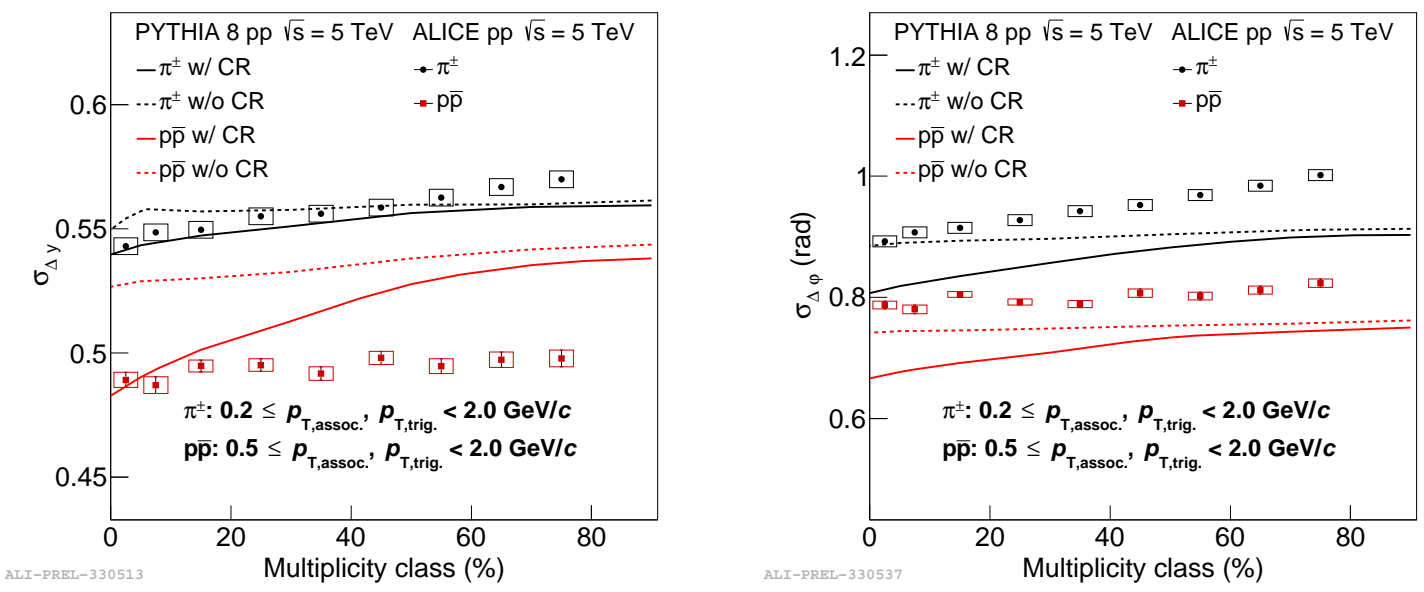

Figure 2: The balance function width in $\Delta y$ (left) and $\Delta \varphi$ (right) for pion and proton pairs as a function of multiplicity class in pp collisions at $\sqrt{s}=5.02 \mathrm{TeV}$.

\subsection{Anisotropic flow and $\mathrm{p}_{\mathrm{T}}$ spectra of identified hadrons}

Recently, a real-valued $v_{2}\{4\}$ was extracted for the first time in pp collisions in ALICE [16]. In addition, non-zero values of $v_{2}\{6\}$ were reported in $\mathrm{p}-\mathrm{Pb}$ and $\mathrm{pp}$ collisions. Such observations 
suggest the presence of long-range multi-particle correlations and can be interpreted as a consequence of the formation and hydrodynamic evolution of a QGP medium in small systems. This interpretation is supported by the fact that identified particle transverse momentum spectra in $\mathrm{p}-\mathrm{Pb}$ and pp collisions show the same characteristic features as in $\mathrm{Pb}-\mathrm{Pb}$ collisions, i.e. a significant hardening with increasing multiplicity more pronounced for heavier hadrons [17]. The latest measurement of $p_{\mathrm{T}}$ spectra of identified particles in $\mathrm{p}-\mathrm{Pb}$ collisions at $\sqrt{s_{N N}}=8.16 \mathrm{TeV}$ confirms this dependence, explained by radial flow effects in nucleus-nucleus collisions. Furthermore, particle ratios such as $p / \pi$ and $\Lambda / K_{S}^{0}$ in p-Pb and pp collisions, reported lately by ALICE in [18] and [19], show qualitatively similar enhancement at intermediate $p_{\mathrm{T}}$ as in $\mathrm{Pb}-\mathrm{Pb}$ collisions [20], attributed to collective motion or, alternatively, quark coalescence at hadronization. The comparison of data with predictions from different models in pp collisions shows only the qualitative agreement most of the times [19].

\section{Conclusions}

In this overview, new studies performed by the ALICE collaboration in $\mathrm{p}-\mathrm{Pb}$ and $\mathrm{pp}$ collisions were discussed. Among these studies, the balance function measurement for identified particles in pp collisions can be highlighted. Since the data are not in agreement with the expectation based on how radial flow should affect particle species with different mass, this leads to a conclusion that collectivity might not be the full explanation behind the narrowing of the balance function width with increasing multiplicity. Such conclusion is strengthened by the fact that neither of PYTHIA8 tunes is able to describe the data. This result, as well as the rest of presented measurements can therefore provide further constrains for current models with the purpose of finding the common theoretical framework for a simultaneous description of various experimental observables and subsequent understanding of collective effects in small systems.

\section{References}

[1] W. Busza, K. Rajagopal, W. van der Schee, Heavy Ion Collisions: The Big Picture, and the Big Questions, Ann.Rev.Nucl.Part.Sci. 68 (2018) 339-376 [hep-ph/1802.04801]

[2] U. W Heinz, R. Snellings, Collective flow and viscosity in relativistic heavy-ion collisions, Ann.Rev.Nucl.Part.Sci. 63 (2013) 123-151 [nucl-th/1301.2826]

[3] X. Zhu et al (ALICE Collaboration), Two-particle correlations in pp and Pb-Pb collisions with ALICE, Int.J.Mod.Phys.Conf.Ser. 29 (2014) 1460212 [hep-ex/1311.2394]

[4] S. Chatrchyan et al (CMS Collaboration), Observation of long-range near-side angular correlations in proton-lead collisions at the LHC, Phys.Lett.B 718 (2013) 795-814 [nuclex/1210.5482]

[5] V. Khachatryan et al (CMS Collaboration), Observation of Long-Range Near-Side Angular Correlations in Proton-Proton Collisions at the LHC, JHEP 09 (2010) 091 [hep-ex/1009.4122]

[6] F. Gelis, E. Iancu, J. Jalilian-Marian, R. Venugopalan, The Color Glass Condensate, Ann.Rev.Nucl.Part.Sci. 60 (2010) 463-489 [hep-ph/1002.0333]

[7] Z.W. Lin, C.M. Ko, B.A. Li, B. Zhang, S. Pal, A Multi-Phase Transport Model for Relativistic Heavy Ion Collisions, Phys.Rev.C 72 (2005) 064901 [nucl-th/0411110] 
[8] A.O. Velasquez, P. Christiansen, E.C. Flores, I. M. Cervantes, G. Pai, Color reconnection and flow-like patterns in pp collisions, Phys.Rev.Lett. 111 (2013) 4, 042001 [hep-ph/1303.6326]

[9] K. Aamodt et al (ALICE Collaboration), Harmonic decomposition of two-particle angular correlations in Pb-Pb collisions at $\sqrt{s_{N N}}=2.76 \mathrm{TeV}$, Phys.Lett.B 708 (2012) 249-264 [nuclex/1109.2501]

[10] B. Abelev et al (ALICE Collaboration), Long-range angular correlations of $\pi, K$ and $p$ in $p$-Pb collisions at $\sqrt{s_{N N}}=5.02 \mathrm{TeV}$, Phys.Lett.B 726 (2013) 164-177 [nucl-ex/1307.3237]

[11] C. H. Christensen, J. J. Gaardhoje, K. Gulbrandsen, B. S. Nielsen, C. Sogaard, The ALICE Forward Multiplicity Detector, Int.J.Mod.Phys.E 16 (2007) 2432-2437 [nucl-ex/0712.1117]

[12] S. Chatrchyan et al (CMS Collaboration), Multiplicity and transverse momentum dependence of two- and four-particle correlations in pPb and PbPb collisions, Phys.Lett.B 724 (2013) 213-240 [nucl-ex/1305.0609]

[13] G. Aad et al (ATLAS Collaboration), Observation of long-range elliptic azimuthal anisotropies in $\sqrt{s}=13 \mathrm{TeV}$ and $2.76 \mathrm{TeV}$ pp collisions with the ATLAS detector, Phys.Rev.Lett. 116 (2016) 17, 172301 [hep-ex/1509.04776]

[14] J.Adam et al (ALICE Collaboration), Multiplicity and transverse momentum evolution of charge-dependent correlations in pp, $p$-Pb, and Pb-Pb collisions at the LHC, Eur.Phys.J.C 76 (2016) 2, 86 [nucl-ex/1509.07255]

[15] A. Buckley et al, General-purpose event generators for LHC physics, Phys.Rept. 504 (2011) 145-233 [hep-ph/1101.2599]

[16] S. Acharya et al (ALICE Collaboration), Investigations of Anisotropic Flow Using Multiparticle Azimuthal Correlations in pp, $p-\mathrm{Pb}, \mathrm{Xe}-\mathrm{Xe}$, and $\mathrm{Pb}-\mathrm{Pb}$ Collisions at the LHC, Phys.Rev.Lett. 123 (2019) 14, 142301 [nucl-ex/1903.01790 ]

[17] B. Abelev et al (ALICE Collaboration), Multiplicity Dependence of Pion, Kaon, Proton and Lambda Production in p-Pb Collisions at $\sqrt{s_{N N}}=5.02 \mathrm{TeV}$, Phys.Lett.B 728 (2014) 25-38 [nucl-ex/1307.6796]

[18] B. Abelev et al (ALICE Collaboration), Multiplicity Dependence of Pion, Kaon, Proton and Lambda Production in p-Pb Collisions at $\sqrt{s_{N N}}=5.02 \mathrm{TeV}$, Phys.Lett.B 728 (2014) 25-38 [nucl-ex/1307.6796]

[19] S. Acharya et al (ALICE Collaboration), Multiplicity dependence of light-flavor hadron production in pp collisions at $\sqrt{s}=7 \mathrm{TeV}$, Phys.Rev.C 99 (2019) 2, 024906 [nucl-ex/1807.11321]

[20] S. Acharya et al (ALICE Collaboration), $K_{S}^{0}$ and $\Lambda$ production in $\mathrm{Pb}-\mathrm{Pb}$ collisions at $\sqrt{s_{N N}}=$ 2.76 TeV, Phys. Rev. Lett. 111 (2013) 222301, 024906 [nucl-ex/1307.5530] 